\title{
A THEOREM OF AHLFORS FOR HYPERBOLIC SPACES
}

BY

\author{
SU-SHING CHEN
}

\begin{abstract}
L. Ahlfors has proved that if the Dirichlet fundamental polyhedron of a Kleinian group $G$ in the unit ball $B^{3}$ has finitely many sides, then the normalized Lebesgue measure of $L(G)$ is either zero or one. We generalize this theorem and a theorem of Beardon and Maskit to the $n$-dimensional case.
\end{abstract}

1. Introduction. In [1], Ahlfors has proved the following:

THEOREM (AHLFORS). If the Dirichlet fundamental polyhedron of a Kleinian group $G$ in the unit ball $B^{3}$ has finitely many sides, then the normalized Lebesgue measure of the limit set $L(G)$ is either zero or one.

He has also conjectured that the limit set $L(G)$ of a finitely generated Kleinian group has the normalized Lebesgue measure either zero or one. In [5], Greenberg has proved that a finitely generated group $G$ can have an infinitely many sided fundamental polyhedron. In this paper, we prove the Ahlfors theorem for $n$-dimensional hyperbolic space under a slightly weaker condition than the finiteness of sides of the fundamental polyhedron.

THEOREM. Let $G$ be a discrete group of isometries of the n-dimensional hyperbolic space $H^{n}$. If the measure of the set of approximation points is greater than the measure of the set of nonapproximation points, then either the measure of $L(G)$ is zero or $L(G)$ is the whole boundary $\partial H^{n}$ of $H^{n}$.

COROLLARY. If $G$ has a finitely many sided convex fundamental polyhedron, then either the measure of $L(G)$ is zero or $L(G)$ is the whole boundary $\partial H^{n}$ of $H^{n}$.

ReMARK. From the above theorem, we see that the set of approximation points is manageable. Only the parabolic fixed points among the nonapproximation points are well known to us. If the group $G$ has a set of nonapproximation points of positive measure, then the Ahlfors theorem fails for that group. According to [2], the limit set $L(G)$ of a purely loxodromic

Received by the editors February 24, 1976 and, in revised form, June 15, 1977.

AMS (MOS) subject classifications (1970). Primary 22E40.

Key words and phrases. Hyperbolic space, Kleinian group, limit set.

๑ American Mathematical Society 1978 
Kleinian group $G$ contains only points of approximation. Thus the limit set of a degenerate, purely loxodromic, Kleinian group is of measure zero.

In [2], Beardon and Maskit have proved the theorem above essentially for $n=3$. They have also extended the Hedlund lemma [8] to $n=3$. Furthermore the converse of the Hedlund lemma is proved. We shall extend this fundamental lemma of Hedlund-Beardon-Maskit to the general $n$-dimension. The proof follows the one of Beardon and Maskit. We do not work on the converse of the lemma, since we only want to prove the Ahlfors theorem. The proof of the theorem of this paper is different from the one by Beardon and Maskit. We use harmonic functions and the Fatou's theorem on radial limits of Knapp [7]. Thus, we give an extension of Ahlfors' original proof. The only merit perhaps is that this approach is more suitable for higher dimensional situations. The approach of Beardon and Maskit is closely related to the metrical part of diophantine approximation and does not seem to be extendable. For other rank one symmetric spaces, this theorem makes sense also [3].

2. Hyperbolic space and Moebius group. The hyperbolic space is the simply connected, complete, Riemannian manifold of constant sectional curvature -1 . There are several models of that space. The unit ball model is $B^{n}=\{x$ $\left.\in \mathbf{R}^{n}|| x \mid<1\right\}$ with the Riemannian metric

$$
d s^{2}=4 \sum_{i=1}^{n} d x_{i}^{2} /\left(1-|x|^{2}\right)^{2} .
$$

The upper half space model is $H^{n}=\left\{x \in \mathbf{R}^{n} \mid x_{n}>0\right\}$ with the Riemannian metric

$$
d s^{2}=\sum_{i=1}^{n} d x_{i}^{2} / x_{n}^{2} .
$$

These two models will be used simultaneously in the sequel.

A Moebius transformation of $\overline{\mathbf{R}^{n}}=\mathbf{R}^{n} \cup\{\infty\}$ with a fixed point in $\overline{\mathbf{R}^{n}}$ can be classified by their conjugacy classes in the group of all Moebius transformations. The canonical forms are as follows:

(1) $x \rightarrow r T x$ with $r \neq 0,1$ and $T \in O(n)$ is loxodromic,

(2) $x \rightarrow T x$ with $T \in O(n)$ is elliptic,

(3) $x \rightarrow T x+a$ with $a \in \mathbf{R}^{n}, T \in 0(n), T(a)=a \neq 0$ is parabolic. A parabolic element which is conjugate to $x \rightarrow x+a$ is called strictly parabolic.

A Moebius transformation of $\mathbf{R}^{n-1}$ has an unique extension to a Moebius transformation of $\overline{\mathbf{R}^{n}}$ which is an isometry of $H^{n}$. Conversely, each isometry of $H^{n}$ is such an extension. The Brouwer fixed point theorem implies that each Moebius transformation of $H^{n}$ has a fixed point in $\overline{\mathbf{R}^{n}}$. Thus, isometries of $H^{n}$ are classified as the above classification of Moebius transformations. 
3. Limit set and convex fundamental polyhedron. Let $G$ be a group of isometries of $B^{n}$ (or $H^{n}$ ). A point $y \in S^{n-1}$ (or $\mathbf{R}^{n-1} \cup\{\infty\}$ ) is called a limit point of $G$ if there is a point $x$ in $B^{n}$ (or $H^{n}$ ) and an infinite sequence $\left\{g_{k}\right\}$ of distinct elements of $G$ such that $y=\lim g_{k}(x)$. The limit set $L(G)$ is the set of all limit points of $G$ and is independent of the choice of $x$. The complement $O(G)$ of $L(G)$ is called the ordinary set of $G$.

A convex fundamental polyhedron $P$ for a discrete group $G$ is an open, convex subset of $B^{n}$ (or $H^{n}$ ) such that

(1) No two points of $P$ are equivalent under $G$.

(2) Every point of $B^{n}$ (or $H^{n}$ ) is equivalent under $G$ to some point of $\bar{P}$.

(3) The sides of $P$ are pairwise identified by elements of $G$.

(4) Every $x$ in $B^{n}$ (or $H^{n}$ ) has a neighborhood that meets only finitely many translates of $P$.

The Dirichlet fundamental polyhedron $P_{0}$ is a convex fundamental polyhedron and is formed as follows. Let $0 \in B^{n}$ be not fixed by any element of $G$. For each $g \in G$, we form

$$
Q_{g}=\left\{y \in B^{n} \mid d(y, 0) \leqslant d(y, g(0))\right\} .
$$

Then $P_{0}=\cap_{g} Q_{g}$ is called the Dirichlet fundamental polyhedron.

For any polyhedron $P \subset B^{n}, \bar{P}$ is the relative closure of $P$ in $B^{n}$ and $P^{*}$ is the intersection of $S^{n-1}$ with the closure of $P$ in $\overline{B^{n}}=B^{n} \cup S^{n-1}$.

A point $z$ of approximation of a discrete group $G$ is a limit point that if $\sigma$ is a geodesic in $B^{n}$ ending at $z$, there is a compact subset $K$ of $B^{n}$ and a sequence $\left\{g_{n}\right\}$ of $G$ such that $g_{n}(\sigma) \cap K \neq \varnothing$. We may take $K=\{x \in$ $\left.B^{n} \mid d(x, 0) \leqslant \rho_{0}\right\}$ and $T=\left\{x \in B^{n} \mid d(x, \sigma) \leqslant \rho_{0}\right\}$. Then $z$ is a point of approximation if and only if $g_{n}(x) \rightarrow z$ in $T$ for some $x$ in $K$ [2].

Proposition 3.1. If $z$ is a fixed point of the loxodromic element $g \in G$, then $z$ is a point of approximation. If $z$ is the fixed point of a parabolic element of $G$, then $z$ is not a point of approximation.

Proof. If $z$ is a fixed point of the loxodromic element $g$ whose axis of translation is $\sigma_{g}$, then $g^{k}(x) \rightarrow z$ in $T .^{1}$ If $z$ is the fixed point of a parabolic element $g$ of $G$, then $g$ preserves the horosphere at $z$. Thus the convergence to $z$ cannot take place in some $T$.

Proposition 3.2. A point of approximation $z$ of $G$ cannot lie on the boundary of a convex fundamental polyhedron $P$ of $G$.

Proof. Since $P$ is convex, we take a geodesic $\sigma$ joining $x$ in $P$ to $z$. By definition, $g_{n}(\sigma) \cap K \neq \varnothing$ for a sequence $\left\{g_{n}\right\}$ and a compact subset $K$. That

${ }^{1}$ Here, inf $\sigma_{g} d\left(x, \sigma_{g}\right)<\rho_{0}$ and $T=\left\{x \in B^{n} \mid d\left(x, \sigma_{g}\right)<\rho_{0}\right\}$. 
is, some point in $B^{n}$ has a neighborhood that meets infinitely many translates of $\sigma$ in $P$. This is impossible.

4. The Hedlund-Beardon-Maskit lemma. The identification of the sides of $P$ induce an equivalence relation on $\bar{P}$ and on $P^{*}$. For each $z \in P^{*}$, the set of points equivalent to $z$ is called the cycle at $z$.

Proposition 4.1. Let $P$ be a convex fundamental polyhedron for $G$ with finitely many sides, then every point of $P^{*}$ is either in $O(G)$ or is a parabolic fixed point.

Proof. Consider $z \in P^{*}$ and conjugate so that $z=\infty$ and the elements of $G$ act on $H^{n}$. Choose $g_{1}, \ldots, g_{r}$ in $G$ so that the cycle of $\infty$ on $P^{*}$ is $\left\{g_{0}(\infty), g_{1}^{-1}(\infty), \ldots, g_{r}^{-1}(\infty)\right\}$, where $g_{0}$ is the identity.

Let $G_{\infty}$ be the stabilizer of $\infty$ in $G$ and $G_{\infty}^{\prime}$ the subgroup of parabolic elements that fix $\infty$. If $\infty \in g\left(P^{*}\right)$, where $g \in G$, we can construct a geodesic $\sigma$ from a point in $g(P)$ to $\infty$. This implies that for some $i, 0 \leqslant i \leqslant r, g_{i} g^{-1}(\sigma)$ is a geodesic ending at $\infty$ and so $g_{i} g^{-1} \in G_{\infty}$. We have $g \in G_{\infty} \cup G_{\infty} g_{1}$ $\cup \cdots \cup G_{\infty} g_{r}$. Furthermore, $G_{\infty}=G_{\infty}^{\prime} \cup G_{\infty}^{\prime} e_{1} \cup \cdots \cup G_{\infty}^{\prime} e_{s}$, where $e_{1}, \ldots, e_{s}$ are elliptic elements. If $G_{\infty}^{\prime}$ is trivial, then a neighborhood of $\infty$ in $\overline{H^{n}}$ meets only a finite number of images of $P$ and so, $\infty \in O(G)$. If $G_{\infty}^{\prime}$ is not trivial then $\infty$ is a parabolic fixed point.

THEOREM 4.1 (HedLUND-BeARDon-MASKit). If there is a convex fundamental polyhedron $P$ for a discrete group $G$ of isometries of $B^{n}$ with finitely many sides, then every limit point of $G$ is either a point of approximation or is a parabolic fixed point.

Proof. Assume that 0 is contained in $P$. Let $z \in S^{n-1}$, and let $\sigma$ be the geodesic from 0 to $z$. If $\sigma$ intersects only finitely many translates of sides of $P$, then for some $g \in G, g(z) \in P^{*}$, and either $z \in O(G)$ or $z$ is a parabolic fixed point.

Now, assume that $\sigma$ passes through infinitely many translates of some side $M$ of $P$. Then $z \in L(G)$. There is a sequence $\left\{g_{n}\right\}$ of $G$ and there is a sequence of points $\left\{y_{n}\right\}$ on $M$ so that $g_{n}(\sigma) \cap M=\left\{y_{n}\right\}$. Assume that $\lim y_{n}=y$. If $y \in B^{n}$, then $z$ is a point of approximation. If $y \notin B^{n}$, then $y$ is a parabolic fixed point. Let $y=\infty$ and consider $H^{n}$. Let $G_{\infty}$ be the stabilizer of $\infty$. The horospheres at $\infty$ are isometric to $\mathbf{R}^{n-1}$. There are two cases: (1) $\mathbf{R}^{n-1} / G_{\infty}$ is compact. The strictly parabolic subgroup $G_{\infty}^{\prime}$ of $G_{\infty}$ is free abelian of rank $n-1$ and is a normal subgroup of finite index in $G_{\infty}$. (2) $\mathbf{R}^{n-1} / G_{\infty}$ is not compact. After a possible conjugation of $G_{\infty}$ by an isometry of $\mathbf{R}^{n-1}, G_{\infty}$ has a normal subgroup $G_{\infty}^{\prime}$ of finite index which is free abelian of rank $k$. Write $\mathbf{R}^{n-1}=\mathbf{R}^{k}+\mathbf{R}^{n-k-1}$. If $g \in G_{\infty}$, then the corresponding Moebius transformation is $A(x)=T x+a$, where $T$ preserves $\mathbf{R}^{k}$ and 
$\mathbf{R}^{n-k-1}$ and $a \in \mathbf{R}^{k} .\left.A\right|_{\mathbf{R}^{k}}$ is strictly parabolic.

In the first case, there is a compact set $K^{\prime} \subset \mathbf{R}^{n-1}$ so that for every $z^{\prime}$ in $\mathbf{R}^{n-1}$, there is an element $P \in G_{\infty}$ with $p\left(z^{\prime}\right) \in K^{\prime}$. Hence, we can choose a sequence $\left\{p_{n}\right\}$ of $G_{\infty}$ so that $p_{n} \cdot g_{n}(z) \in K^{\prime}$ and $p_{n} \cdot g_{n}(0) \rightarrow \infty$. The latter implies that infinitely many of the $\left\{p_{n} \cdot g_{n}\right\}$ are distinct. Since the geodesics of $H^{n}$ are circles and straight lines perpendicular to $\mathbf{R}^{n-1}$, it is easy to find a compact subset $K$ in $H^{n-1}$ which intersects $p_{n} \cdot g_{n}(\sigma)$. Thus $z$ is a point of approximation.

In the second case, since $T$ is in $O(k) \times O(n-k-1)$, every ball $B_{t}$ in $\mathbf{R}^{n-k-1}$ is preserved by $T$. Consequently, the complement $U$ of it is also preserved. When $n=3$, this complement is the cusped region $U=\left\{z|| I_{m} z \mid\right.$ $\geqslant t$ ] [2]. We may assume that no translates of $z$ lie in $U$ by taking a suitable radius of the ball $B_{t}$. Then we may pick a sequence $\left\{p_{n}\right\}$ in $G_{\infty}$ so that $p_{n} g_{n}(z) \in K^{\prime} \times B_{t}$ where $K^{\prime}$ is a compact set in $\mathbf{R}^{k}$. By the same hyperbolic geometric consideration, $z$ is a point of approximation.

5. The Ahlfors theorem. We need the following theorem of Fatou type which is proved by Knapp [7] on radial limits of harmonic functions.

THEOREM 5.1 (KNAPP). Let $H$ be a noncompact symmetric space of rank one. If $f$ is a function in $L^{1}(\partial H)$, where $\partial H$ denotes the boundary of $H$, and if $h$ is its Poisson integral

$$
h(x)=\int_{\partial H} P(z, x) f(z) d z,
$$

where $d z$ denotes the normalized Lebesgue measure on $\partial H$, then $h$ is harmonic and the radial limit $\lim _{t \rightarrow \infty} h(\sigma(t))$, along a geodesic $\sigma$ ending at $z$, converges to $f(z)$ almost everywhere with respect to the given measure.

THEOREM 5.2. Let $G$ be a discrete group of isometries of $B^{n}$. Suppose that the set of nonapproximation points of $L(G)$ has Lebesgue measure less than that of the approximation points. Then either $L(G)$ is $S^{n-1}$ or $L(G)$ has zero Lebesgue measure.

Proof. Let $f$ be the characteristic function of $L(G)$ in $S^{n-1}$. Then the Poisson integral of $f$ is a harmonic function

$$
h(x)=\int_{S^{n-1}} P(z, x) f(z) d z,
$$

where $d z$ denotes the normalized Lebesgue measure on $S^{n-1}$. Moreover, $0 \leqslant h(x) \leqslant 1$ for all $x$ in $B^{n}$. By Theorem 5.1, the radial limit of $h(x)$ exists and almost everywhere equals to $f(z)$, for $z \in S^{n-1}$. It is easy to see that $h$ is $G$-invariant, because $L(G)$ is $G$-invariant and hence $f(z)$ is $G$-invariant. If $L(G)$ is $S^{n-1}$, then there is nothing to show. Assume that $L(G)$ is a proper subset of $S^{n-1}$. If $L(G)$ has Lebesgue measure zero then there is nothing to 
show. If $L(G)$ has positive measure, then there must be a point $z$ of approximation in $L(G)$ such that the radial limit of $h(x)$ along a geodesic $\sigma$ approaching to $z$ must exist and equal to 1 . There is a sequence $\left\{z_{n}\right\}$ of points in $\sigma$ approaching to $z$ such that there are elements $\left\{g_{n}\right\}$ in $G$ and $g_{n}\left(z_{n}\right)$ is in a compact set $K$. There is a subsequence of $\left\{g_{n}\left(z_{n}\right)\right\}$ approaching to a point $x$ in $K$. By continuity and $G$-invariance, $h(x)=1$. But 1 is the upper bound for $h(x)$. Hence $h(x)$ attains its maximum at an interior point $x$ in $B^{n}$. Thus $h(x)$ has to be the constant function 1 . Therefore the measure of $L(G)$ is

$$
m(L(G))=\int_{L(G)} P(z, x) d z=h(x)=1 .
$$

COROLlaRY. If $G$ is a discrete group of isometries of $B^{n}$ such that its Dirichlet fundamental polyhedron has finitely many sides, then $L(G)$ is either $S^{n-1}$ or of Lebesgue measure zero. If $G$ is of the second kind, then $L(G)$ has Lebesgue measure zero.

Proof. According to Theorem 4.1, $L(G)$ consists of approximation points or parabolic fixed points. But the set of parabolic fixed points is countable and hence is of Lebesgue measure zero.

\section{REFERENCES}

1. L. V. Ahlfors, Fundamental polyhedrons and limit point sets of Kleinian groups, Proc. Nat. Acad. Sci. U.S.A. 55 (1966), 251-254.

2. A. Beardon and B. Maskit, Limit points of Kleinian groups and finite-sided fundamental polyhedra, Acta Math. 132 (1974), 1-12.

3. S. Chen and L. Greenberg, On hyperbolic spaces, Contributions to Analysis, Academic Press, New York, 1974.

4. L. Greenberg, Finiteness theorems for Fuchsian and Kleinian groups, Lecture Notes, LMSNATO Conference on Discrete Groups and Automorphic Functions, Cambridge, England, Summer, 1975.

5. __ Fundamental polyhedra for Kleinian groups, Ann. of Math. (2) 84 (1966), 433-441.

6. Discrete subgroups of the Lorentz group, Math. Scand. 10 (1962), 85-107.

7. A. W. Knapp, Fatou's theorem for symmetric spaces. I, Ann. of Math. (2) 88 (1968), 106-127.

8. J. Lehner, Discontinuous groups and automorphic functions, Math. Surveys, no. 8, Amer. Math. Soc., Providence, R. I., 1964.

9. N. Weilenberg, Discrete Moebius groups, fundamental polyhedra and convergence, Dissertation, University of Minnesota, 1974.

Department of Mathematics, University of Florida, GatNesViLle, Florida 32611 